SHORT REPORT

\title{
The geography of the highest mortality areas in Spain: a striking cluster in the southwestern region of the country
}

\author{
J Benach, Y Yasui, J M Martínez, C Borrell, M I Pasarín, A Daponte
}

Occup Environ Med 2004;61:280-281. doi: 10.1136/oem.2002.001933

\begin{abstract}
Aims: To determine the areas of Spain with the most increased mortality risk.

Methods: Age adjusted relative risk of death by gender was estimated in each of 2218 small areas of the country using a non-parametric empirical Bayes method. To determine areas with "significantly increased risk" a Poisson based score test was used.

Results: Mapping of the highest risk areas showed a striking geographical clustering in the southwestern region of the country. This region, comprising $8 \%$ of the Spanish population, accounts for about one third (2884 deaths) of the total excess mortality.
\end{abstract}

M ortality inequalities across geographical localities have been well documented in various developed countries. ${ }^{1}$ Our research in Spain has shown that mortality inequalities at the small area level are a widespread phenomenon. ${ }^{2}$ Here we report a striking observation in Spain that the areas with the highest increased risk of death are clustered in one specific region of the country. This presents a pressing public health concern with respect to the country's health policy and calls for attention from researchers and policy makers.

\section{METHODS}

To assess the mortality risk by locality, we estimated age adjusted relative risk of death by gender in each of the 2218 small areas of Spain established in our previous research. ${ }^{3}$ To estimate the relative risk in each small area we obtained, by age and gender, all-cause mortality counts aggregated for a nine year period (1987-95) and the 1991 census population counts. Based on these data, age-gender specific mortality rates of Spain were estimated by a Poisson regression model with the generalised estimating equation (GEE) modification to account for within-area correlation of mortality across ages and genders. ${ }^{4}$ These age-gender specific mortality rates of Spain were used as "reference rates" for calculating expected

\section{Main messages}

- Small area studies are a valuable tool to identify areas with the highest mortality.

- Mapping of the highest risk areas revealed a striking geographical clustering in the southwestern region of Spain.

- These high risk areas have a statistically significant increased risk of death which imposes a large public health burden. counts of death by gender in each small area. The relative risk in each small area was estimated by a non-parametric empirical Bayes method for a standardised mortality ratio (SMR), the ratio of observed versus expected counts of death. ${ }^{5}{ }^{6}$ The comparison of SMRs across small areas assumes a uniform rate ratio across age-sex strata within each small area. We used the SMR approach under this required assumption, instead of the direct standardisation approach, because stratum specific rates were unstable in our small areas and the application of the direct standardisation was untenable.

To determine small areas with "significantly increased risk" we tested the null hypothesis of the age adjusted relative risk equalling 1.0 by a Poisson based score test that takes into account the estimated amount of overdispersion from the GEE model. Let $\mathrm{O}$ and $\mathrm{E}$ be the observed and expected deaths in each small area and $\phi$ be the estimate of the overdispersion parameter based on the Poisson assumption. The significance test statistic is defined as: $\mathrm{T}=(\mathrm{O}-$ $\mathrm{E})^{2} / \phi \mathrm{E}$, which is compared to a $\chi^{2}$ distribution with one degree of freedom. The small area was considered as a significantly increased risk area if the departure from the null hypothesis was statistically significant at the $5 \%$ level and the non-parametric empirical Bayes estimate of the relative risk was above 1. Within the set of the significantly increased risk areas, we have only mapped the areas with the "highest risk" defined as being above the median relative risk among the significantly increased risk areas.

\section{RESULTS}

The total number of deaths in the whole country was 2958041 in the nine year period. The areas with the highest risk were $8 \%(n=177)$ and $10 \%(n=219)$ of all areas for men and women, respectively. Mortality relative risk estimates of those areas ranged from 1.19 to 1.89 for men (median 1.22) and from 1.19 to 1.61 (median 1.24) for women. Annual excess mortality, defined as the difference between the number of observed and expected deaths, accounted for 5197 deaths in men and 3602 deaths for women in the areas with the highest risk.

\section{Policy implications}

- Results suggest the need for in-depth multidisciplinary investigations of various potentially damaging environmental, occupational, and social risk factors on mortality.

- Findings have important implications for health policy, suggesting that high risk areas should be the highest priority of public health policies in Spain. 


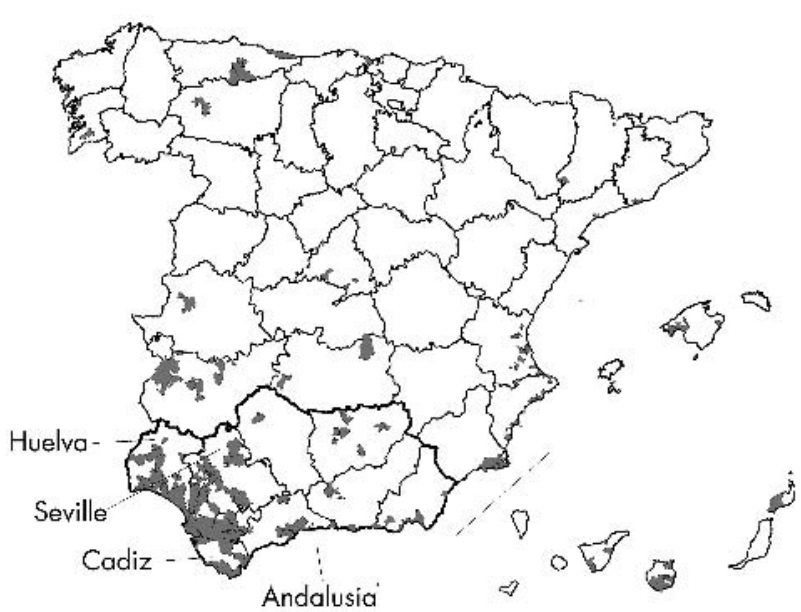

Figure 1 Areas with the highest mortality risk among men.

Mapping of the highest risk areas reveals a striking geographical clustering in the southwestern region of the country. For men, half of the areas $(n=88)$ with the highest mortality levels are located in the southern region of Andalusia; a third $(n=60)$ are in the southwest provinces of Huelva, Seville, and Cadiz (fig 1). For women, a little less than half of the areas $(\mathrm{n}=98)$ are in the region of Andalusia; a quarter $(n=56)$ are located in the same three provinces (fig 2). Among the 152 small areas located within the three provinces, 60 areas (40\%) for men and 56 areas (37\%) for women are in the highest mortality levels. Huelva, Sevilla, and Cadiz, comprising $8 \%$ of the Spanish population, account for about one third (2884 deaths) of the total excess mortality.

\section{DISCUSSION}

The clustering of the worst mortality areas in the southwestern region of Spain, especially in the provinces of Huelva, Seville, and Cadiz, for both men and women, is consistent and astonishing. The data reveal that these "dangerous" areas have a statistically significant increased risk of death that imposes a large public health burden which urgently demands attention and action.

This result raises a number of important research questions: What does it mean to have a regional clustering of such worst areas? What is the significance of both men and women sharing many of the worst areas in the same region? Why can such clustering occur? This analysis does not show the causes of this clustering of increased mortality. Some evidence exists, however, that in some of these areas there is a high level of damaging environmental (for example, river transported heavy metals and urban air pollutants), ${ }^{7-9}$ occupational (for example, asbestos), ${ }^{10}$ and social factors (for example, unemployment and overcrowding $)^{2}$ which may increase the risk of death. Our results should lead to in-depth multidisciplinary investigations of various individual and contextual potentially important environmental, occupational, and social risk factors on mortality. In addition, the results also have important implications for health policy, suggesting that these mapped high risk areas should be the highest priority of social and public health policies in Spain. Researchers and policy decision makers are called to pay attention to these findings.

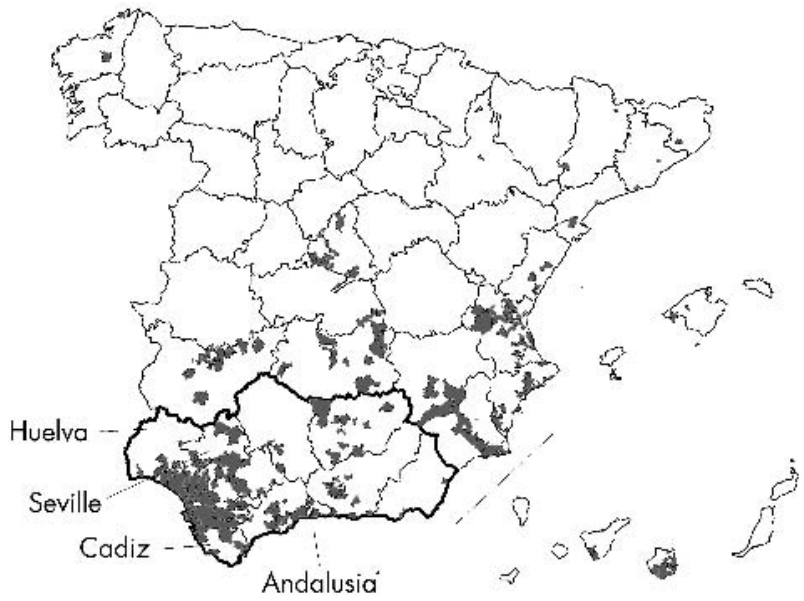

Figure 2 Areas with the highest mortality risk among women.

\section{Authors' affiliations}

Joan Benach, Department of Experimental Sciences and Health, Occupational Health Research Unit, Universitat Pompeu Fabra, Barcelona, Spain

Y Yasui, Cancer Prevention Research Program, Fred Hutchinson Cancer Research Center, Seattle, USA

J M Martínez, Department of Experimental Sciences and Health, Occupational Health Research Unit, Universitat Pompeu Fabra, Barcelona, Spain

C Borrell, Agency of Public Health of Barcelona, Spain M I Pasarin, Agency of Public Health of Barcelona, Spain A Daponte, Andalusian School of Public Health, Granada, Spain

This study was partly funded by a grant from a Research Grant Programme on Health Targets from MSD

Correspondence to: Dr J Benach, Universitat Pompeu Fabra, Department of Experimental Sciences and Health, Occupational Health Research Unit, Carrer Dr Aiguader 80, 08003 Barcelona, Spain; joan.benach@upf.edu

Accepted 24 February 2003

\section{REFERENCES}

1 Carstairs V. Socio-economic factors at area level and their relationship with health. In: Elliott P, Wakefield JC, Best NG, Brigss DJ, eds. Spatial epidemiology. Methods and applications. Oxford: Oxford University Press, 2000.

2 Benach J, Yasui Y, Borrell C, et al. Material deprivation and leading causes of death by gender: evidence from a nation-wide small-area study. J Epidemiol Community Health 2001;55:239-45.

3 Benach J, Yasui Y, Borrell C, et al. Atlas of mortality in small areas in Spain (1987-1995). Barcelona: UPF/MSD, 2001

4 Liang KY, Zeger SL. Longitudinal data analysis using generalized linear models. Biometrika 1986;73:13-22.

5 Böhning D. Computer-assisted analysis of mixtures and applications metaanalysis, disease mapping, and others. Boca Raton and London: Chapman Hall, 1999.

6 Yasui Y, Hao L, Benach J, et al. An empirical evaluation of various priors in the empirical Bayes estimation of small-area disease risks. Stat Med 2000;19:2409-20

7 Ruiz F. Trace metals in estuarine sediments from the southwestern Spanish coast. Mar Pollut Bull 2001:42:482-90.

8 Scientific Research Superior Council (CSIC). Primer informe del estudio que coordina el Consejo Superior de Investigaciones Científicas sobre el diagnóstico ambiental y sanitario de la ría de Huelva, 2001. [First report of the study coordinated by the Scientific Research Superior Council on the environmental and sanitary diagnose of the Huelva's estuary]. http:// www.CSIC.es/prensa/RiaHuelva (accessed 8 November 2001).

9 Alcaraz Pelegrina JM, Martinez-Aguirre A. Natural radioactivity in groundwaters around a fertilizer factory complex in South of Spain. Appl Radiat Isot 2001;55:419-23.

10 Escolar Pujolar A, Gonzaléz A, Agudo A, et al. Information about occupational exposure to asbestos given to cases in an etiological study: ethical aspects. Eur J Epidemiol 2001;17:1-6. 